American Journal of Economics and Business Administration 2 (4): 366-376, 2010

ISSN 1945-5488

(C) 2010 Science Publications

\title{
Market and Economic Regulation Periodicity: A Feature that Allows Prevention and Regulation
}

\author{
Laure Jehlen \\ Department of Finance, Dauphine University, F-75015, \\ CEREG DRM 2002-9, Paris
}

\begin{abstract}
Problem statement: This study addressed the essential questions of non linear financial flows forecasting and prevention regarding the means of periodicity. Approach: We implemented some new computational tools to weigh up the presence of periodicity in 10 years financial price time series. The data consists of NYSE-Euronext listed corporations' closing prices and East-Asian, NorthAmerican and European markets indexes closing prices. Results: Empirical evidence put forward that the tools allow considering the periodicity plus a forecasting side effect for the next months. The lengths of the cycles are determined for indexes and assets with strong disparity between the two categories. Conclusion: Extensively, the results open the road to determine structural changes in time series by allowing the calculation of the fundamental frequencies. It introduces some concepts from physics and discusses their potential usefulness in financial economics. This shows the way to detect financial and economic extreme events (burst of bubbles, companies' failures, markets or sectors' recessions) as to prevent them.
\end{abstract}

Key words: Financial markets, international finance forecasting and simulation, financial crises, NLSE, periodicity

\section{INTRODUCTION}

Kendall and Hill (1953); Osborne (1962); Cootner (2000) and Mandelbrot (1962) investigated the statistical properties of the successive prices of shares, their unpredictable character and the shape of their distributions of probability. They all agree to say that there are no doubts on the presence of periodicity in the financial time series. Granger and Joyeux (1980), as well as Hosking (1981); Granger (1995) and Levy (1954), developed long dependence stochastic models. The most fervent opponent, Lo (1991), countered his own results explaining that the results could be bound to the method he used: "If there is long dependence in the returns, the accurate test is still missing to prove it". Facing this "no evidence" proof, we search to assess this periodicity through methodological investigations.

Specifically, the aim was to confirm the necessary condition of periodicity that allows modeling the market place prices global evolution by means of the NLSE (a nonlinear non quantum Schroedinger equation). For this purpose, we have developed two methods that will be discussed in this study: One through fundamental frequencies computation, the other by means of polynomial fit. Both methods are based on the adaptation of the Von Neuman spectral theorem, through respectively Fejer theorem and Weierstrass theorem, to the model Jehlen (2009a; 2009b). We discovered that most importantly, the second method allows smoothing and denoizing of the financial price time series. Hence, it facilitates the research for the harmonics. These are the fundamental frequencies playing a role in interferences and extreme events occurrence.

In the second part, we summon up the assumptions and the theory of the model Jehlen (2009a; 2009b). Despite the successes in the past of the models modeling the Brownian motion by means of the one dimensional linear heat equation and assets returns dynamics by means of diffusion processes resulting from the Gaussian and one-dimensional framework of Black and Scholes (1973) and Merton (1973) (solved by Merton by means of the heat equation), we broaden these models to consider a non linear and twodimensional framework and show that both reasoning and mathematics ensue then in the Schrodinger equation. The reasoning and the mathematics allowing us to switch to the Schrodinger equation are wholly and extensively developed in our previous study (Jehlen $2009 a ; 2009 b)$. The objective of the current study is not any longer to prove the new fundamental model developed. On the one hand, it consists in explaining the quantitative methods that had to be determined and implemented to put in evidence the periodicity in the 
financial assets prices time series and on the other hand in sharing the forecasting helpfulness of the resulting experimentations with the research community.

The third part presents the materials and methods dedicated to validate the periodicity condition for the assets prices trajectories. Two methodologies are implemented assessing the periodicity of market prices time series and by consequence the use of the NLSE (Nonlinear Schrodinger Equation) for modeling packets of market prices trajectories. The two inventive methodologies are explained theoretically and experimented on ten years time series of real data, on the one hand, daily closing prices of six international indexes from 1993-2003 and on the other hand, daily closing prices of companies listed on Euronext from 1995-2005 and belonging to the equipment economic sector. The fourth part shows that the findings corroborate perfectly the assumptions, showing the way to detect burst of bubbles, companies' failures, markets or sectors' recessions as to prevent them. It describes also the different contributions and achievable applications of the new model and finally, the fifth part concludes.

\section{Theoretical background:}

The fundamentals of the model and its postulates: The description of the considered system: The marketplace is thought about as a whole dynamic system in evolution (Fig. 1). The marketplace capitalization is the middle in which the studied asset price dynamics take place. The assets prices evolve in an environment having its own characteristics: the stock market where their quotation takes place. In our model, the whole marketplace, its indexes and shares and the amount of money which is exchanged and invested there, constitute the system.

The asset prices and the total value of the capitalization of the marketplace are the studied random variables.

Figure 1 shows the target representation to be reached by applying the model. All the securities price trajectories of a whole marketplace are put one to one by front of waves to form the envelope of the marketplace value and volume evolution.

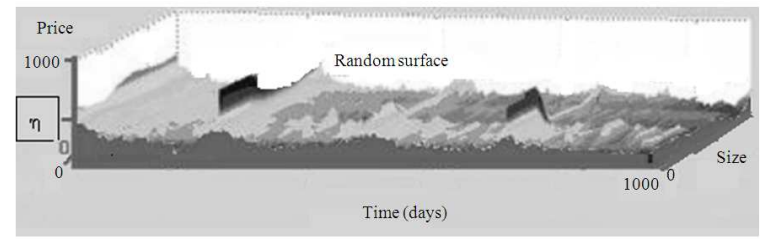

Fig. 1: Representation of a market place as a dynamic system
As the surface consists of several component waves, of various periods and wavelengths, the rise of the level of the marketplace, the free surface $\eta$ is the geometrical sum of the constituents of the waves. The vertical axis represents by projection the value of the prices evolution for every listed company on the marketplace. The Fig. 1 represents a double vertical axis: The upper positive vertical axis, the above zero semi-axis represents the price elevation or decline of each traded assets on the market place (represented by the random surface) at each time and the negative vertical axis, the below zero semi-axis represents the depth under the surface envelope. The $X$ axis represents the time t. The $Y$ axis represents the additive part of each security in the total capitalization of the marketplace. The size of a security represents its proportion in the total capitalization.

The postulates and features of the model Jehlen (2009a; 2009b): Before reminding here the main assumptions and equations of the model built in these previous studies, we should specify that it is not simply an application of new PDEs taken from physics. It consists in the result of numerous years of research for extending the fundamental one-dimensional heat equation and Gaussian framework for the representation equations of asset returns dynamics into a twodimensional and non linear representation of a market place assets prices dynamics.

The first postulate: It states the necessary existence of two strengths components of the dynamics. Therefore the model postulates two necessary strengths for the asset prices motion. These two strengths allow the asset value to oscillate and pass by equilibrium phases. The diffusion strength results from the shocks due to the sell and buy orders of the operators on the marketplace. It is the diffusion without jumps commonly considered in diffusion process modeling. Taking in account a potential strength is new. This potential strength depends on the position of the asset price, it represents the endogenous characteristics of the share (and furthermore of the listed company). We mean by endogenous characteristics what makes the firm's value (potential of growth, fundamental value, total of assets, turnover, market share, capital, reputation) of the firm. This potential component introduces the existence of a "sleeping" intrinsic nonlinearity.

The conjecture of the existence of two strengths components implies that the future value of the studied random variables depends on the position, $X=(x, t, z)$, at the time of measure $t$ and on the period of time $\tau$ between the last measure and the future measure. We 
define the position $\mathrm{X}$ by the following parameters: $\mathrm{x}$ is the size of the asset in the capitalization of the marketplace at the time of measure $t ; z$, is the asset price at the time of measure $t$; $t$ is the time of measure.

The studied variables, the asset prices and the total value of the capitalization of the marketplace, are supposed continuous and differentiable functions of (x, $\mathrm{t}, \mathrm{z})$.

The second postulate: The property of flow for the considered trajectories induces the choice of the Linear Schrodinger Equation (LSE) as basic representation equation: This postulate allows us to define the basic equation of the built model. The detailed proof of the property of flow for financial asset price time series is given in Jehlen (2009b).

Chorin and Marsden (1993) provide the following definition of a flow: "A flow is the propagation, according to time, of a countable quantity (mass, volume, energy), or uncountable quantity (wave), in an environment". Considering our model, we observe that the collection of all the future possible states (possible asset prices future positions) is uncountable.

The Fig. 2 represents the bundle of possible paths between two measures $M$ and $M$ ' of an asset position $\mathrm{X}$.

The set of possible future values and positions is uncountable; therefore the future trajectories can be modeled as waves. However the observations of asset prices positions (the realizations) belong to a real and compact domain taking values in $\mathrm{R}^{3}$. As a result the studied system is also limited to a bounded region of the phase space, besides it presents a fractal character. The detailed mathematical proof (Jehlen, 2009a; 2009b) confirms that the representation equation for the dynamics of the prices of the packets of assets traded on a market place develops too in the linear Schroedinger equation, SLE.

The specific LSE of the model spells:

$$
\frac{\partial \Phi}{\partial \mathrm{t}}=-\mathrm{a} \frac{\partial \Phi}{\partial \mathrm{x}}+\mathrm{i} \alpha \frac{\partial^{2} \Phi}{\partial \mathrm{x}^{2}}
$$

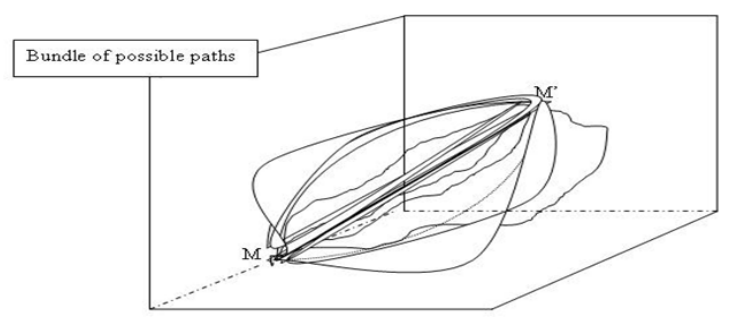

Fig. 2: Representation of the bundle of possible paths where, a, $\alpha$, are real coefficients; $\Phi$ (the flow field function), is a complex function of $\mathrm{x}$ (the position) and $\mathrm{t}$ and $i^{2}=-1$. Thus, the model allows the representation of the dynamics of prices composing the random envelope (surface) engendered by the packets of assets listed on the marketplace, by means of the SLE, (when extreme events do not intervene).

The third postulate: This postulate adjusts the model to take in account extreme events occurrences. It assumes that the origin of the extreme events (even if connected to an intrinsic structural defect, (Sornette, 2009; Aglietta, 2008), embedded in the potential, (Jehlen, 2009a), is triggered by an important external non linearity.

This postulate allows us to add a non linear term, $\mathrm{i} \beta|\Phi|^{2} \Phi$, to the LSE equation for the general representation of the dynamics of the assets. This term represents the external instabilities at the origin of crashes, bubbles and consequent corrections of prices (the massive contributions and removing of capital, the excessive speculations and the contagions). We postulate that it can either be positive or negative. The associated risks belong to the systemic risks. The specific Nonlinear Schrodinger Equation for our model spells:

$\frac{\partial \Phi}{\partial \mathrm{t}}=-\mathrm{a} \frac{\partial \Phi}{\partial \mathrm{x}}+\mathrm{i} \alpha \Delta \Phi+\mathrm{i} \beta|\Phi|^{2} \Phi$

It is denoted NLSE. Where a, $\alpha, \beta$ are real coefficients; $\Phi$, the flow field function, is a complex function of $x$ (the position) and $t$; and $i^{2}=-1$. Hence, the financial assets price series are supposed non linear, nor stationary, but periodic.

Since Bachelier (1995) and Black and Scholes (1973) and Merton (1973), denoted 1973 BSM, every continuous time default risk model or pricing model considers the Wiener Brownian as a determinant of the asset dynamics equation. Einstein (1905), characterizes the Brownian motion by the means of the heat equation as representation equation of the dynamics. He considers a perfect fluid and the normality of the distribution of the moves. Merton (1973) assumes the form $\mathrm{C}(\mathrm{S}, \mathrm{t})=$ $f(t) y\left(u_{1}, u_{2}\right)$, for the solution of the BSM PDE and represents the dynamics of the unknown function $y$ by means of the one-dimensional equation of heat, denoted EH. The assumptions are perfect market hypotheses and gaussianity of the distribution of the returns.

The Table 1 establishes the correspondences between Merton (1973) and Einstein (1905) and our upper-dimensional model in accordance with Jehlen (2009a; 2009b). 
Am. J. of Economics and Business Administration 2 (4): 366-376, 2010

Table 1: Correspondences between Merton (1973); Einstein (1905) and Jehlen (2009a, 2009b) models

\begin{tabular}{lll}
\hline Merton (1973) & Einstein (1905) & Jehlen (2009a; 2009b) \\
\hline $\begin{array}{l}\text { Solving of the European call } \\
\text { price 1973 BSM PDE by means of the EH }\end{array}$ & $\begin{array}{l}\text { EH representation for the dynamics } \\
\text { of the Brownian motion }\end{array}$ & $\begin{array}{l}\text { LSE representation of the dynamics of packets } \\
\text { of assets prices, out of extreme events occurrence }\end{array}$ \\
$\frac{\partial \mathrm{y}}{\partial \mathrm{u}_{2}}=\frac{1}{2}\left(\frac{\partial^{2} \mathrm{y}}{\partial^{2} \mathrm{u}_{1}}\right)$ & $\frac{\partial \mathrm{f}}{\partial \mathrm{t}}=\mathrm{D} \frac{\partial^{2} \mathrm{f}}{\partial \mathrm{x}^{2}}$ & $\frac{\partial \Phi}{\partial \mathrm{t}}=-\mathrm{a} \frac{\partial \Phi}{\partial \mathrm{x}}+\mathrm{i} \alpha \frac{\partial^{2} \Phi}{\partial \mathrm{x}^{2}}$ \\
Constant coefficient $1 / 2$ & $\begin{array}{l}\text { Constant diffusion coefficient D } \\
\text { depending on the middle }\end{array}$ & $\begin{array}{l}\text { Real coefficients } a \text { and } \alpha \\
\text { depending on the market place }\end{array}$ \\
Function y & Flow function f & $\begin{array}{l}\text { Envelope flow field function } \Phi \\
\text { Variable } \mu_{2} \text { (Time t) }\end{array}$ \\
Variable $\mu_{1}$ & Time t & Time t \\
One-dimensional frame & Spatial coordinate x & Spatial position $\mathrm{X}=(\mathrm{x}, \mathrm{t}, \mathrm{z})$ \\
\hline
\end{tabular}

Periodicity, a feature that allows forecasting and prevention, analysis and regulation: The following results ensue from the necessity to verify the conditions of non stationary, periodicity and size) allowing to apply the NLSE equation. We will see that the compliance to the size condition is obvious. Non stationary has largely been put in evidence in financial literature. The third condition, periodicity, until today was more controversy, periodicity was assumed in consideration of long dependence but cycles had never been measured.

\section{MATERIALS AND METHODS}

The data considered for the operated tests: The data used for these tests are, the daily closing prices from 1993 till 2003 of the French CAC 40, Canadian TSX, German DAX, English FTSE, American S\&P500 and the Japanese NIKKEI, indexes and the quotations on Euronext from 1995 till 2005 of French companies of the specific branch of industry motor and equipment and automotive. This chosen sector consists of 213 companies. It represented in $2005,36.7 \%$ of the turnover and $20 \%$ of the added value of the total manufacturing industry, as well as $16.4 \%$ of the workforce, what makes it interesting to study. The dataset of Euronext quotations originates from the laboratory DRM-CEREG CNRS, UMR 7088 at ParisDauphine University, with Euronext-Parisbourse Limited Company sourcing. The high frequency dataset volume for the 86 listed securities represents a total of 23 gigabytes. For the indexes, historical data constitute a base of 5.1 gigabytes. The size of the dataset files made complex their manipulation and required doublecore computer equipment and a Linux universe for their treatment. To fulfill the testing of the periodicity we decided to consider only the daily closing prices. The daily closing prices from 1995 till 2005 for the companies securities consist of 210000 observations and the indexes empirical analysis is founded on 175 900 daily closing prices, over a period ranging from
April 27th, 1993 to July 14th, 2003, for the six indexes considered. All the companies in the 213 panel show the first year a turnover superior to 750000 Euros and leverage superior to 350000 Euros. This excludes from the dataset the tiny companies but ensures a rather homogeneous sampling. The tests are based on the calculation specificities and programming (explained pages 5 to 9 in this study when we describe the methods) of Matlab blocksets and toolboxes provided by the Mathworks company.

The conditions of appliance of NLSE and the periodicity: To apply the NLSE equation three conditions must be satisfied: The size of the elements moving in the middle must be tiny in consideration of the volume of the middle. The time series must be non stationary and periodic.

As shown in Jehlen (2009a; 2009b), the condition of size is satisfied for the indexes as well as the asset prices. For the indexes, the size of an individual share represents in average $10^{-9}$ of the capitalization of the index and respectively for the companies, the nominal of an individual share of the sector represents in average $1.12 \times 10^{-9}$ of the total float of the sector.

The condition of non stationary characteristic of asset price time series is also satisfied. It has been largely proven in the financial literature. The non stationary has been demonstrated by numerous authors, from Mandelbrot (1971); Boness et al. (1974); Fielitz (1971); Lo and MacKinlay (1988) and Poterba and Summers (1988), to McCauley (2004) stating: "Observed financial market distributions are very far from stationary".

By consequence, the key point consists in assessing the condition of periodicity.

The literature point of view on periodicity: Though there are no doubts about the existence of periodicity in financial asset price time series, the computational evidence is still missing. Facing this "no evidence" 
assertion, we search to assess this periodicity feature through some new methodological investigations.

In the early sixties, Kendall and Hill (1953); Osborne (1962); Cootner (2000) and Mandelbrot (1962) investigated the statistical properties of the successive prices of shares, their unpredictable character and the shape of their distributions of probability. They all agree to say that there are no doubts on the presence of periodicity in the financial time series. Mandelbrot (1963) states that periodicity may not be detected as the period of the phenomenon exceeds the interval of measure. Then the period extends over all the interval of measure, rejecting the no periodicity postulate. We will see in the results part that the cycles that we put in evidence for the assets daily closing prices time series are effectively large.

The Fig. 3 in the vein of Von Arx, (1962), is an example of periodic phenomenon for which the period extends beyond the range of measure: It represents the movements of the water of a lake and the periodic cycle of the move.

Since the eighties, numerous researchers have linked periodicity with non stationary and long range dependence evidence as Greene and Fielitz (1977). Granger and Joyeux (1980), as well as Hosking (1981) and Granger (1995), developed long dependence stochastic models. The most fervent opponent, Lo (1991), countered his own results explaining that the results could be bound to the method he used: "if there is long dependence in the returns, the accurate test is still missing to prove it".

The new periodicity tests developed for our model: If asset prices dynamics behave like waves there must exist fundamental frequencies characterizing their periodicity. This study implements two innovative methods of periodicity characterization for financial time series. We illustrate these two methods on both the real data of daily closing prices for the indexes and for the securities belonging to the studied economic sector.

First method, the frequencies determination by means of the spectral Fejer theorem for the computation of the fundamental frequencies: The principle of calculation for the search for periodicity is straightforward, the following example Andreasen (2005) explains it easily: "If we are interested in the measure of the temperature in a given place according to time, intuitively we can expect to have a dominant frequency of $1 / 24 \mathrm{~h}$ or 0,042 which represents the maximum of heat in the daytime (peak at around midday) and the minimum of heat at night (hollow towards midnight). By considering an interval of a week, we can approximate the temperature variations according to time by a sinusoidal wave having a period of $24 \mathrm{~h}$. If the Fourier Transform is made on this sinusoidal wave, it is noticed that it contains only a single frequency".

Of course, this case is elementary and needs no special calculation method to help at the determination of the period. It is totally different in the case of the financial prices time series, we study. Then methods like the Fourier Transform or the Fast Fourier Transform are of great help. The advantage of the Fast Fourier Transform is that it separates the even frequencies of the odd frequencies during the calculation of the Discrete Fourier Transform to decrease the number of operations. Thus we characterize the harmonics (fundamental frequencies) by means of the Fourier decomposition theorem, the calculation of the Fourier coefficients and the determination of the spectral Density of Power specified either in days per cycles or in frequencies, the one being the inverse of the other.

The first step of reasoning and computation is the following: The Fourier decomposition in harmonic waves allows a fine analysis of the non stationary signals. It is based on the seminal works of Huygens (1690), those of Bernoulli (1754) and those of Fourier (1768-1830). Huygens (1690), defined the famous principle of overlapping: "The waves cross each other and unite so that, appreciably, they form a single wave. The wave, in a given point, is the overlapping of the wavelets emitted by the diverse points". This principle can be considered as "the" principle constituent of the concept of wave. If a simple addition allows combining waves, then reciprocally these waves can be decomposed into a sum of elementary waves, called harmonics.

Second, the Fourier Theorem allows to formalize mathematically this decomposition: It reads for real functions: «Any function $\mathrm{f}(\mathrm{x})$ of a real variable $\mathrm{x}$ can under certain conditions of regularity, that we shall suppose satisfied, be decomposed into a sum of harmonics of the variable $\mathrm{x}$, according to $f(x)=\sum_{n} C_{n} \exp \left[i\left(\omega_{n} x+\alpha n\right)\right]$. Each elementary Fourier coefficient is characterized by its rate of evolution $\omega$ in $\mathrm{x} »$. In continuous time, these discrete sums must, naturally, be replaced by an integral in $\omega$.

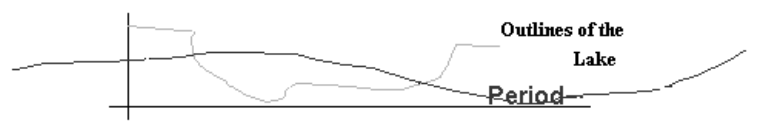

Fig. 3: The period of the move extends the range of measure (the lake) 
Third, as we model financial asset prices time series like waves, we base the method on the trigonometric Fejer theorem (the extension of the Fourier theorem for complex valued functions): "Let denote $f$, (in our testing: The function of each asset prices path), a complex valued function, supposed periodic and defined over the period $\mathrm{T}$, (in our testing: 10 years) and let $C_{n}$ be the associated series of the Fourier coefficients, $C_{n}=\int_{T} f(t) \exp (-2 \pi$ int $) d t$, then the function $f(t)$ can be described as a suite of cosine and sine functions on the interval $(0, T)$ of module $C_{n}$. For $\mathrm{n}=0$, the value of the coefficient of Fourier is the mean value of $f$.

The second method is the non parametric polynomial fit of the studied time series: It is based on the extension of the Von Neuman spectral theorem for our studied series: "Let $f(t)$, (our function of asset prices path), be a continuous function on an interval as a Banach space (the path we consider is continuous and the measures (the realizations) belong to a compact domain of $\mathrm{R}^{3+}$ ), then according to the theorem of Weierstrass, it can be found a suite of polynomials $\operatorname{Pn}(t)$ approaching $\mathrm{f}(\mathrm{t})$ uniformly on this interval”.

LEMMA: "First, let us consider the possible future trajectories of the prices for the built model, the possible future states of the system are modeled by a process denoted $\Phi(\mathrm{X}, \mathrm{t})$ which takes value in the vector-space defined by a base of $\mathrm{N}$ vectors: $\Phi(\mathrm{X}, \mathrm{t}): \Omega \times[0,+\infty[\rightarrow \mathrm{M}$ where the set $\mathrm{M}$ is defined by: $\mathrm{M} \equiv\left\{\overrightarrow{\mathrm{e}}_{1}, \ldots, \overrightarrow{\mathrm{e}}_{\mathrm{N}}\right\} \subset \mathrm{R}^{\mathrm{N}}$, under real probability and where $\mathrm{X}$ is the position that we defined previously as $\mathrm{X}$ $=(\mathrm{x}, \mathrm{t}, \mathrm{z})$, at the time $\mathrm{t}$.

Second, in contrast, the collection of the observed values (asset prices positions) of the studied dynamic system is measurable. These realizations belong to a compact subset $\beta$ of $\mathrm{M}$. Then by application of the theorem of Heine, $\beta$ being a compact subset of this normed vector space, any continuous function on $\beta$ is uniformly continuous there.

Third, considering the functional that are the linear continuous applications composing the trajectories, these linear continuous applications of the space into itself form a Banach space, consequently the spectral theorem applies to the studied financial series".

\section{RESULTS}

For the first method: The Computation of the Fourier coefficients is operated for the 86 companies and the six indexes by use of MATLAB for the programming of the algorithm. In this phase, the output is a set of complex numbers defining the amplitude and the phase of the frequencies components that shows symmetry near the imaginary axis $\mathrm{I}_{\mathrm{m}}=0$.

For instance, the two Fig. 4 and 5 display the coefficients resulting in the case of respectively the company 29512 and of the French index CAC 40.

We operate the calculation of the periodogram that is a technique of estimation of the spectral density of power of the signals, by use of MATLAB for the programming of the algorithm, for each index and companies time series. This spectral density captures the frequency content of the stochastic process and helps identify the periodicities. The spectral density of power is computed as the square of the magnitude of the Fourier transform of the signal.

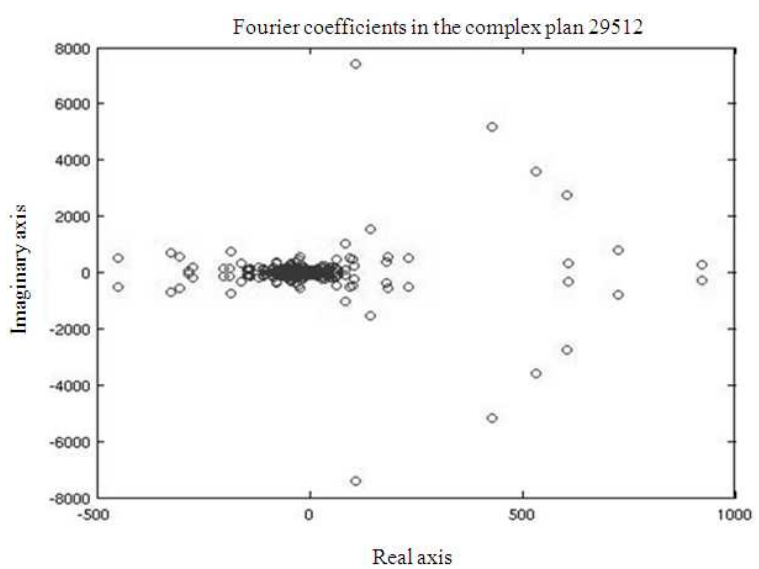

Fig. 4: Fourier coefficients for the company 29512

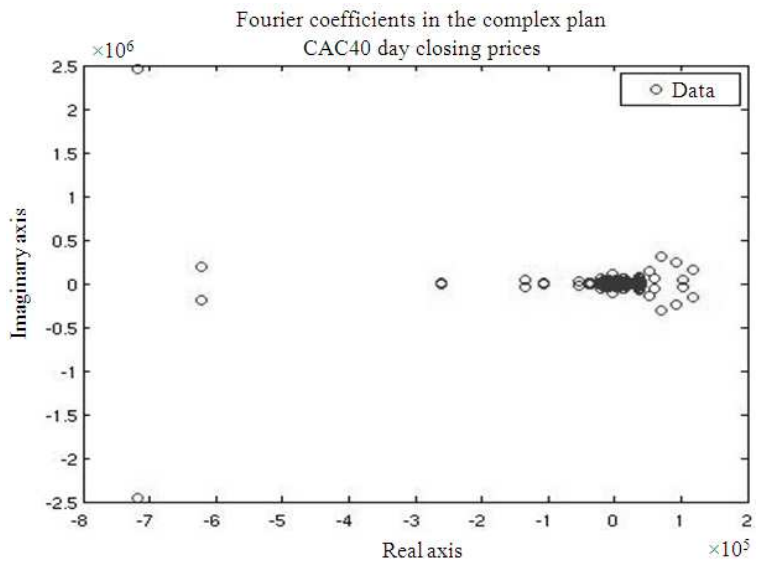

Fig. 5: Fourier coefficients for the index CAC40 
Am. J. of Economics and Business Administration 2 (4): 366-376, 2010

Table 2: Summary of the diverging range features of cycles for companies and indexes

\begin{tabular}{llllllll}
\hline Closing prices & CAC 40 France & NIKKEI Japan & TSX Canada & DAX Germany & Company 29512 & Company 35356 Company 43625 \\
\hline Cycles in & 300 & 300 & $250-300$ & 250 & 120 & 280 & 100 \\
Number of days & 500 & 500 & 600 & 600 & & & \\
& & 800 & & & & & \\
\hline
\end{tabular}

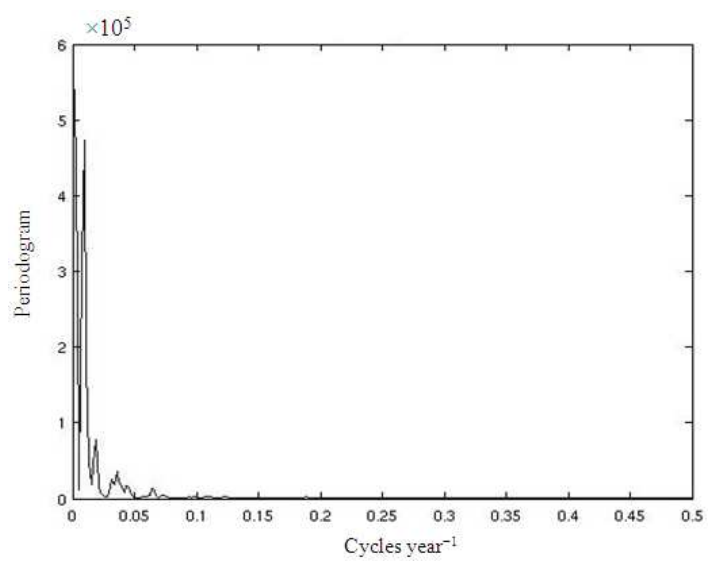

Fig. 6: Power spectrum density of the frequencies for the company 29512

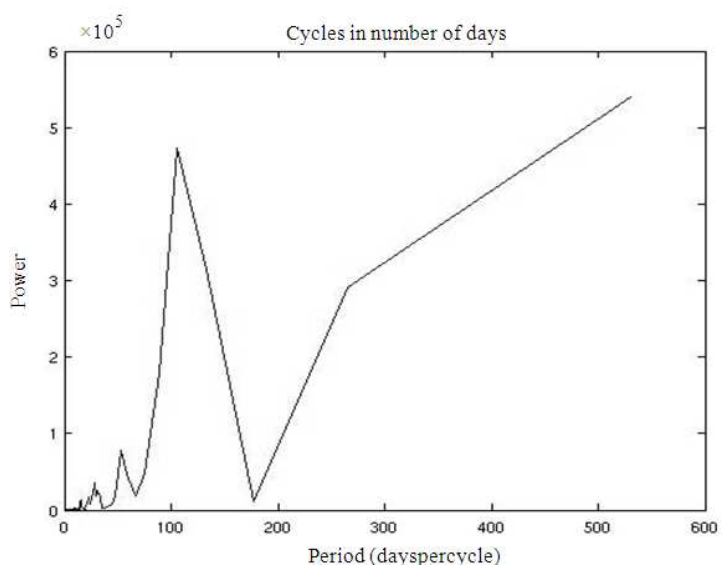

Fig. 7: Power spectrum density of the periods for the company 29512

By use of MATLAB for the programming of the algorithm, we compute the periodograms, plotted in the Fig. 6 and 7, of the power of the signal, in order to respectively render the frequency and the period for the 29512 company.

This method has been implemented on both Indexes and Companies of the data set. The results are summarized in the representative extract of the Table 2.

The results bring to light the periodicity and allow the valuation of the periods and the frequencies. The findings show a large difference between the indexes and the companies periodicity.

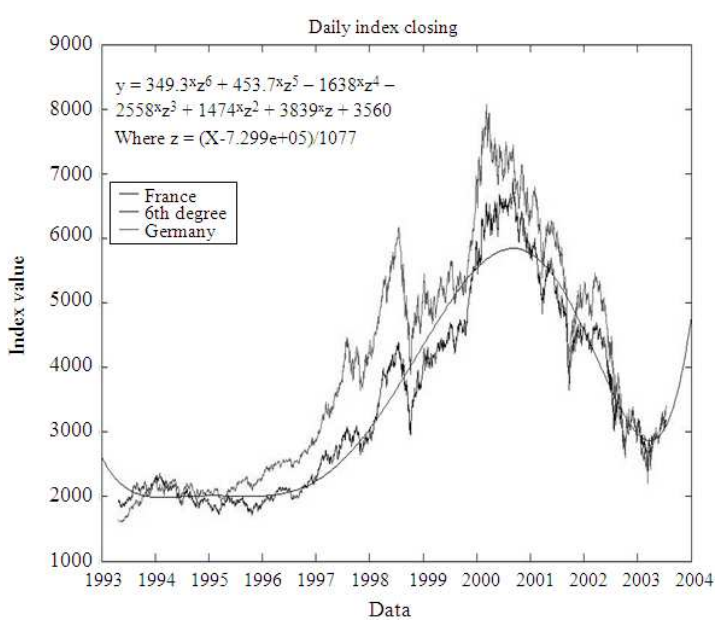

Fig. 8: Polynomial approximation of French CAC 40 index ten years time series

The indexes present generally two cycles lasting around 300 and 500 days and the companies, a unique cycle which is shorter, close to one hundred days. We report also, in this Table 2, two exceptions that are the 35356 company which frequency (280 days) is out of the range for unknown reason and the Japanese NIKKEI Index for which a third cycle intervenes in the series.

For the second method: The method shows predictive results (ranging from 4-6 months), for the values of all the studied assets, for the six international indexes tested, as for all the companies of the sector tested. More importantly, the method allows smoothing the time series to facilitate the research for the harmonics (fundamental harmonics intervening in interferences and extreme events). These outcomes arise while considering either ten years or two years daily closing prices time series, (a complement testing would be to test this feature for other frequencies of measure then daily closing prices).

Only 10 figures are given in this study for preserving the number of pages of the study in reasonable length.

The polynomial fit of the ten years time series of daily closing prices for the French CAC40 index from April 1993 to July 2003 results in the Fig. 8. (The other index time series represented above the CAC 40 Index prices concerns the German index DAX). The fit is the smooth (denoised curve). The Fig. 9 covers the next months of the polynomial estimation from 1987-2007. 


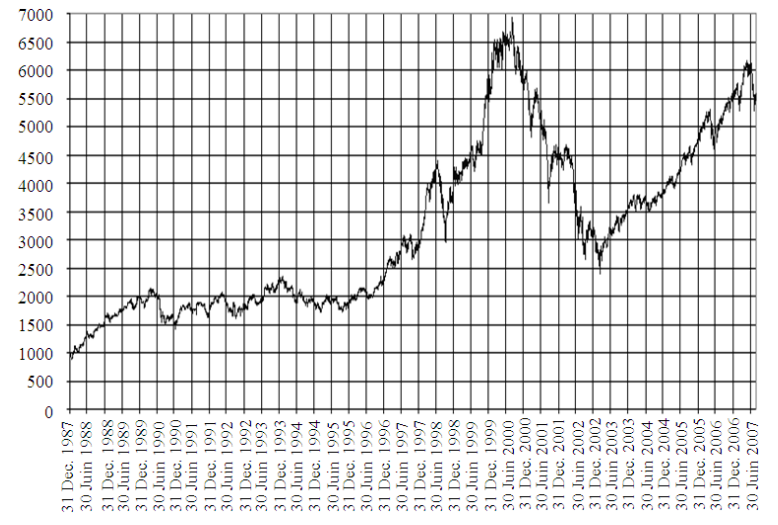

Fig. 9: Twenty years of French CAC 40 index closing prices

It shows the good prediction of the future tendency by the fit and the excellent forecast character of the method on a short-term basis of four to six months.

\section{DISCUSSION}

\section{Consequences of the periodicity feature:}

- The periodicity feature legitimates the use of the NLSE to model market place envelopes

- The periodicity feature allows the detection of interferences and Benjamin Feir instabilities through frequency and speed calculations.

The Benjamin Feir focal interference expresses itself through explosions and collapses. If calculation of speed is trivial, this method allows the determination of the harmonics. Therefore the methods developed in this study help to detect focal interference or absorption of energy from nearby securities and open the way to detect abnormal moves, bubbles and collapses (on-going research).

- The periodicity feature embedded in the studied time series authorizes Haar decomposition of the time series. It allows the detection in the middle of the profusion of the peaks, of the moment at which the trajectories change as well as the amplitude of the structural change

By applying the Haar decomposition to the global set of the 83 companies of the sector, only one of the companies' time series showed a rupture and a change in its structure. The decomposition is plotted in the Fig. 10.

Wavelet analysis has not a predictive effect. However the computation of frequencies and speeds enables to determine the problem before occurrence.
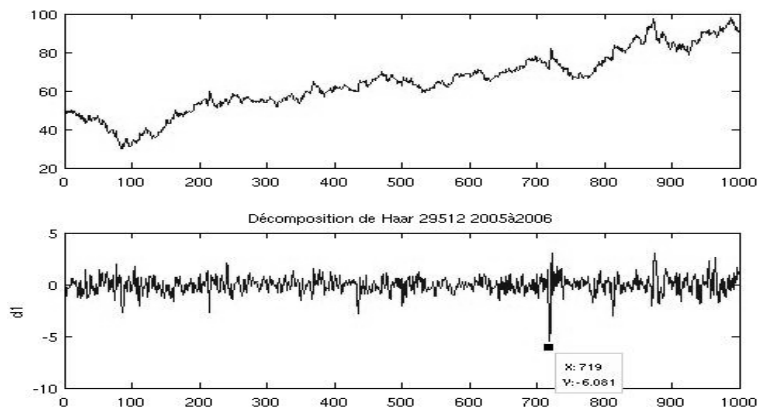

Fig. 10: Haar decomposition of price time series

This periodic feature combined with theoretical mathematical modeling through NLSE computation, (Jehlen 2009a; 2009b), can help at the better comprehension of crisis and ruptures and all the more useful for prevention and regulation.

Researchers in hydrodynamics (Dysthe and Trulsen, 2001), proposed a particular solution of the NLSE, to explain the giant waves of $30 \mathrm{~m}$ height in oceans, causing the wrecks of big ships.

The short-term price evolution forecast feature put in evidence through polynomial fitting. The polynomial fit seems to take on an unmistakable quality of short-term extrapolation of the future values. However, this method was not driven for the forecast of prices but to simplify the search and calculation of the fundamental frequencies. The polynomial fit main quality consists in simplifying the time series into simple cosine and sine curves. It results in a sort of smoothing of the time series oscillations. Thus the determination of the fundamental frequencies of the signals by this second approach can be largely facilitated, the noise being eliminated from the series.

Contribution of the model itself (Jehlen, 2009a; 2009b): By deviating from the Gaussian and onedimensional frame of Black and Scholes (1973), Merton (1973), denoted 1973 BSM, specific applications are: Extending the Black and Sholes (1973) and Merton (1973) frame and endogenous Markov regime-switching models for the calculation of options.

Besides, the global 2D reading of the market place time series aims at detecting the similar behavior of classes of assets, the inflections points and the degrees of development of bubbles before breaks.

NLSE computation may be used to model the evolution in respect to the time of the random surface envelope in order to forecast global price evolution and instabilities. 
The computation of geometric rupture points aims at detecting the instabilities like inflection points even in individual trajectories.

\section{CONCLUSION}

Using data of NYSE-Euronext listed corporations and data of East-Asian, North-American and European markets indexes, we implemented some new computational tools in finance that are harmonics computation by means of the Fourier Decomposition theorem, on the one hand and polynomial fit through Weierstrass theorem, on the other hand. Empirical evidence shows that the harmonics' determination, the cycles' determination and the smoothing of the time series are successful with these tools. For the first time, the periodicity is fully put in evidence in financial asset price time series and the frequencies and the periods are measured. In addition, the method points up the great disparity of indexes' price evolution cycles (250-600 days) and securities' price evolution cycles (100-250 days) for daily closing price time series. Besides, it pinpoints a systematical forecasting side effect for the four to six next month's values. Finally, this study weights up the periodicity assumption of the model we proposed in Jehlen (2009a; 2009b), allowing the modeling of financial prices evolution according to time by means of the NLSE, Nonlinear Schrodinger Equation. Therefore, another input of the study resides in introducing some concepts from physics, to make them known to a wider scientific community and discuss their potential usefulness in financial economics. Hence, it has the potential to stimulate the interest of applied econometricians and financial economists. Extensively, the model intends to find out structural changes in time series through the calculation of the fundamental frequencies. This shows the way to detect financial and economic extreme events (bubbles and burst of bubbles, companies' failures, markets or sectors' recessions, excessive speculation) as to prevent them and for regulation purposes as well (on-going research).

\section{Endnotes:}

- The specific SLE equation does not entail the Planck constant $\hbar=\frac{\mathrm{h}}{2 \pi}$. Our economic frame departs from the quantum framework of the initial equation of Schroedinger describing the motion of an electron of mass $\mathrm{m}$ in a potential $\mathrm{V}$. (i $\left.\hbar \frac{\partial \Phi}{\partial \mathrm{t}}=\frac{-\hbar}{2 \mathrm{~m}} \Delta \Phi+\mathrm{V} \Phi\right)$ as the scale of observation is not the same. Therefore our equations (resembling the Novikov et al. (1984) water wave equations used by Chang et al. (1995), do not entail the Planck constant. That assumption taken in our 20062008 work is also established in Nottale (2009). It relies on the "principle of relativity of scale", which postulates that the fundamental laws of nature must be valid regardless of the "state of scale" system of reference. It complements the "principle of relativity" of Galilean, Poincare and Einstein, which applies to statements of position, orientation and movement. In a fractal space-time as the one we consider for the model, the fundamental law of dynamics in such geometry is especially that of the Schroedinger equation, which can be generalized as to no longer necessarily depend on the microscopic Planck's constant.

- For the same reason as the one given for the specific LSE, there is no Planck constant in the considered NLSE equation

- Former researchers have applied spectral theory and hydrodynamics to finance. By instance, Azais et al. (2005), model the level up crossings with a maximum likelihood method, based on the hypotheses of normality and stationary of the moves. Recently Printems (2005), started studying the stochastic behavior of the Korteweg de Vries equation in the case of the INRIA Premia project, which is dedicated to improve option pricing methods

\section{ACKNOWLEDGEMENT}

The researchers would like to thank Prof. Christopher Hennessy, A. Prof. Alexandros Milionis and Prof Jacques Hamon, for their excellent advice, as well as the conferences and seminar participants at the 6th. International Conference on Applied Financial Economics, Greece, 2009 and for their useful comments and interest, A. Prof. Sebastien Pouget, Toulouse, A. Prof. Virginie Terrazza, Luxembourg. This study has benefited from helpful comments of an anonymous referee and from material funding of MATLAB and Simulink, by The Mathworks Company, France, that are gratefully acknowledged. The usual disclaim applies.

\section{REFERENCES}

Aglietta, M., 2008. La Crise. 1st Edn., Editions Michalon, Paris, ISBN: 9782841864775, pp: 125.

Andreasen, A., 2005. Utilization scientifique de Python-Deuxieme partie: Analyse de donnees. Gazette Linux. http://ftp.traduc.org/doc-vf/gazettelinux/html/2005/115/lg115-A.html 
Azais, J.M., J.R. Leon and J. Ortega, 2005. Geometrical characteristics of Gaussian sea waves. J. Applied Probab., 42: 407-425. DOI: 10.1239/jap/1118777179

Bachelier, L., 1995. Theorie de la Speculation. Theorie Mathematique du Jeu (1900). Editions Jacques Gabay, Paris, ISBN: 2-87647-129-9, pp: 152.

Black, F. and M. Scholes, 1973. The pricing of options and corporate liabilities. J. Polit. Econ., 81: 637-654. http://www.jstor.org/pss/1831029

Bernoulli, D., 1754. Exposition of a new theory on the measurement of risk. Econometrica, 22: 22-36. DOI: $10.2307 / 1909829$

Boness, A.J., A.H. Chen and S. Jatusipitak, 1974. Investigations of non stationary in prices. J. Bus., 47: 518-537.

Cootner, P., 2000. The Random Character of Stock Market Prices. 1st Edn., Risk Books, USA., ISBN: 10: 1899332847 , pp: 618.

Chang, Q., B. Guo and H. Jiang, 1995. Finite difference method for generalized Zakharov equations. Math. Comput., 64: 537-553. http://www.jstor.org/pss/2153438

Chorin, A.J. and J.E. Marsden, 1993. A Mathematical Introduction to Fluid Mechanics. 3rd Edn., Springer, USA., ISBN: 0387979182, pp: 172.

Dysthe, K.B. and K. Trulsen, 2001. The evolution of an evolution equation. Proceedings of the Conference: Progress in Nonlinear Science, (PNS'01), UIO, Nizhny Novgorod, pp: 1-15. http://folk.uio.no/karstent/waves/Nizhnypaper.pdf

Einstein, A., 1905. Uber die von der molekularkinetischen Theorie der Wärme geforderte Bewegung von in ruhenden Flussigkeiten suspendierten Teilchen. Annalen Physik, 17: 549-560. http://www.zbp.univie.ac.at/dokumente/einstein2.p df

Fielitz, B.D., 1971. Stationary of random data: Some implications for the distribution of stock price changes. J. Finan. Q. Anal., 6: 1025-1034. http://www.jstor.org/pss/2329918

Granger, C.W.J. and R. Joyeux, 1980. An introduction to long-memory time series models and fractional differencing. J. Time Ser. Anal., 1: 15-29. DOI: 10.1111/j.1467-9892.1980.tb00297.x

Granger, C.W.J., 1995. Modeling nonlinear relationships between extended-memory variables. Econometrica, 63: 265-279. http://www.jstor.org/stable/2951626
Greene, M.T. and B.D. Fielitz, 1977. Long-term dependence in common stock returns. J. Finan. Econ., 4: 339-349. DOI: 10.1016/0304$405 \times(77) 90006-X$

Hosking, J.R.M., 1981. Fractional differencing. Biometrika, 68: 165-176. DOI: 10.1093/biomet/68.1.165

Huygens, C., 1960. Traite de la lumiere. JOC/EFR. http://www-groups.des.stand.ac.uk/ history/Extras/Huygens_lumiere.html

Jehlen, L., 2009a. Modeling of the financial flows. Proceedings of the 7th ACDD Conference, Apr. 13, Strasbourg, France, pp: 1-50.

Jehlen, L., 2009b. A new nonlinear model on financial price modeling. Proceedings of the 4th FFM Conference on Forecasting Financial Markets, May 27-29, FFM, Luxembourg, pp: 1-25.

Kendall, M.G. and A.B. Hill, 1953. The analysis of economic time-series-part I: Prices. J. R. Stat. Soc. Ser. $\quad$ A, 116: 11-34. http://www.jstor.org/pss/2980947

Levy, P., 1954. Theorie de L'addition des Variables Aleatoires. 2nd Edn., Gauthier-Villars, Paris, ISBN: 2876472074, pp: 416.

Lo, A.W., 1991. Long-term memory in stock market prices. Econometrica, 59: 279-1313. http://www.jstor.org/stable/2938368

Lo, A.W. and A.C. Mackinlay, 1988. Stock market prices do not follow random walks: Evidence from a simple specification test. Rev. Financ. Stud., 1: 41-66. http://www.jstor.org/pss/2962126

Mandelbrot, B., 1962. Statistical dependence in prices and interest rates. National Bureau of Economic Research.

Mandelbrot, B., 1963. The variation of certain speculative prices. J. Bus., 36: 394-419. http://www.jstor.org/stable/2350970

Mandelbrot, B.B., 1971. When can price be arbitraged efficiently? A limit to the validity of the random walk and martingale models. Rev. Econ. Stat., 53: 225-236. http://www.jstor.org/pss/1937966

McCauley, J.L., 2004. Dynamics of Markets: Econophysics and Finance. 1st Edn., Cambridge University Press, Cambridge, ISBN: 13: 9780521824477, pp: 226.

Merton, R.C., 1973. Theory of rational option pricing. Bell J. Econ. Manage. Sci., 4: 141-183. DOI: $10.2307 / 3003143$

Nottale, L., 2009. Des Fleurs Pour Schrödinger: La Relativité D'échelle et ses Applications. 1st Edn., Ellipses Marketing, Paris, ISBN: 978-2729851828, pp: 421. 
Novikov, S., S.V. Manakov, L.P. Pitaevskii and V.E. Zakharov, 1984. Theory of Solitons: The Inverse Scattering Method. 1st Edn., Springer, Moscow, ISBN: 10: 0306109778, pp: 292.

Osborne, M.F.M., 1962. Periodic structure in the Brownian motion of stock prices. Operat. Res., 10: 345-379. http://www.jstor.org/pss/167679

Poterba, J. and L.H. Summers, 1988. Mean reversion in stock returns: Evidence and implications. J. Financ. Econ., 22:

27-59. http://EconPapers.repec.org/RePEc:eee:jfinec:v:22: y:1988:i:1:p:27-59
Printems, J., 2005. Random modulation of multisolitons for the stochastic $\mathrm{KdV}$ equation. Bilan CNRS.

Sornette, D., 2009. Why Stock Markets Crash: Critical Events in Complex Financial Systems. 1st Edn., Princeton University Press, Princeton, ISBN: 9781400829552, pp: 448.

Von Arx, W.S., 1962. Introduction to Physical Oceanography. 1st Edn., Addison-Wesley Publishing Co., Inc., Reading, MA., pp: 422. 